\title{
Bound states in the continuum in graphene quantum dot structures
}

\author{
J. W. González ${ }^{1}$, M. Pacheco ${ }^{1 *}$, L. Rosales ${ }^{2}$ and P. A. Orellana ${ }^{3}$ \\ ${ }^{1}$ Departamento de Física, Universidad Técnica Federico Santa María, Casilla 110 V, Valparaíso, Chile \\ ${ }^{2}$ Instituto de Física, Pontificia Universidad Católica de Valparaíso, Casilla 4059, Valparaíso, Chile and \\ ${ }^{3}$ Departamento de Física, Universidad Católica del Norte, Casilla 1280, Antofagasta, Chile
}

(Dated: August 19, 2018)

\begin{abstract}
The existence of bound states in the continuum was predicted at the dawn of quantum mechanics by von Neumann and Wigner. In this work we discuss the mechanism of formation of these exotic states and the feasibility to observe them experimentally in symmetrical heterostructures composed by segments of graphene ribbons with different widths forming a graphene quantum dot. We identify the existence of bound states in the continuum in these graphene quantum dot systems by means of local density of states and electronic conductance calculations.
\end{abstract}

The new material denominated graphene is a single layer of carbon atoms which can be fabricated by different methods like mechanical peeling or epitaxial growth [2 4]. Nanoribbons are stripes of graphene which can be obtained through high-resolution lithography [5], by controlled cutting processes [6] or by unzipping multiwalled carbon nanotubes [7]. The electronic behavior of all these nanostructures is mainly determined by their geometric confinement which allows the observation of quantum effects such as quantum interference effects, resonant tunneling and localization effects. The possibility to control these quantum effects, by applying external perturbations to the nanostructures or by modifying the geometrical confinement, could be used to develop new technological applications, such as graphene-based composite materials [8], molecular sensor devices [9, 10] and nanotransistors [11].

An interesting feature exhibited by certain confined nanostructures, such as quantum dots systems, is the presence of bound states in the continuum (BICs). Their existence was predicted at the dawn of quantum mechanics by von Neumann and Wigner [12] for certain spatially oscillating attractive potentials, for a one-particle Schrödinger equation. Much later, Stillinger and Herrick 13] generalized von Neumann's work by analyzing a two-electron problem, they found BICs were formed despite the interaction between electrons. The occurrence of BICs was discussed in a system of coupled Coulombic channels and, in particular, in a Hydrogen atom in a uniform magnetic field [14]. BICs have also shown to be present in the electronic transport in mesoscopic structures [15 20]. More recently, exploiting the analogy between electronics and photonics, Marinica et al. 21, Bulgakov and Sadreev [22] and Prodanović et al. 223] reported the presence of BICs in photonic systems. Several mechanisms of formation of BICs in open quantum dots (QDs) have been reported in the literature. The simplest one is based on the symmetry of the systems and, as a consequence, in the difference of parity between the QD eingenstates and the continuum spectrum [24]. Another mechanism takes into account a nonzero coupling between bound states in the QD and the con- tinuum spectra. The formation of BICs would be the result of a destructive interference process of these resonances, for certain variations of the physical parameters of the QD 25 27]. A third mechanism for the BICs formation in optics, is associated with the Fabry-Perot interferometer 30].

Until nowadays, there is only one experimental work, reported by Capasso and co-workers [31], in which BICs were measured in semiconductor heterostructures grown by molecular beam epitaxy. Thereby, the search of new systems which could be able to reveal the existence of BICs, with the possibility to be measured, is a very interesting and relevant field of research. The experimental feasibility exhibits by graphene based systems and the great advances in the controlled manipulation and measurements reported in graphene, together with the possibility of modifying their electronic properties by applying external potentials, suggests that BICs could be observable in graphene quantum dots heterostructures.

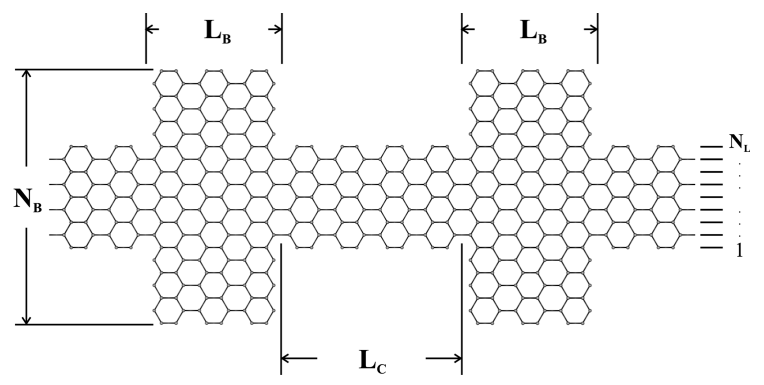

FIG. 1. Schematic view of a GQD structure with leads of width $N_{L}=9$, a conductor region composed by two symmetrical junctions of width $N_{B}=21$ and length $L_{B}=3$ separated by a central structure of length $L_{C}=4$ and width $N_{C}=9$

In this work we study the formation of BICs in quantum-dot like structures, formed by segments of graphene ribbons with different widths connected between each other [33]. We identify the presence of BICs in these symmetrical graphene quantum dots (GQDs) and we discuss the mechanism for their formation. We found that the GQD local density of states as a function of the energy shows the presence of a variety of sharp peaks that 
we demonstrate are BICs. The linear conductance also shows the presence of resonant states which contribute to the electronic transmission. By changing the geometrical parameters of the structure, it is possible to control the number and position of these resonances as a function of the Fermi energy.

A schematic view of the considered systems is presented in figure 1. The conductor is formed by two symmetric crossbar junctions of widths $N_{B}$ and length $L_{B}$, and a central region that separates the junctions, of width $N_{c}$ and length $L_{C}$. Two semi-infinite leads of width $N_{L}=N_{c}$ are connected to the ends of the central conductor. We studied the different electronic states manifested in the system as a function of the geometrical parameters of the GQD structure.

Systems are described by using a single $\pi$-band tight binding Hamiltonian, taking into account first nearest neighbor interactions with a hopping parameter $\gamma_{0}$. We consider hydrogen passivation by setting a different hopping parameter for the carbon dimmers at the ribbons edges [34], $\gamma_{\text {edge }}=1.12 \gamma_{0}$. To calculate electronic properties of the system we adopt the surface Green's functions matching formalism [10, 36]. In this scheme, we divide the heterostructure in three parts, two leads composed by semi-infinite pristine graphene nanoribbons, and the conductor region composed by two nanoribbon crossbar junctions, as it is shown in figure 1 .

In the linear response approach, the electronic conductance is calculated by the Landauer formula. In terms of the conductor Green's functions, it can be written as 37] $G=\frac{2 e^{2}}{h} \bar{T}(E)=\frac{2 e^{2}}{h} \operatorname{Tr}\left[\Gamma_{L} G_{C}^{R} \Gamma_{R} G_{C}^{A}\right]$, where $\bar{T}(E)$, is the transmission function of an electron crossing the conductor region, $\Gamma_{L / R}=i\left[\Sigma_{L / R}-\Sigma_{L / R}^{\dagger}\right]$ is the coupling between the conductor and the respective leads, given in terms of the self-energy of each lead: $\Sigma_{L / R} V_{C, L / R} g_{L / R} V_{L / R, C}$. Here, $V_{C, L / R}$ are the coupling matrix elements and $g_{L / R}$ is the surface Green's function of the corresponding lead [10]. The retarded (advanced) conductor Green's functions are determined by [37] $; G_{C}^{R, A}=\left[E-H_{C}-\Sigma_{L}^{R, A}-\Sigma_{R}^{R, A}\right]^{-1}$, where $H_{C}$ is the Hamiltonian of the conductor.

The Fig 2 displays results of the local density os states (LDOS) (upper panel) and the linear conductance (lower panel) for a GQD structure formed by two armchair ribbons leads of width $N_{L}=5$ and a conductor region composed by two symmetric crossbar junctions of width $N_{B}=17$, length $L_{B}=3$ and relative distances between the junctions $L_{C}=5$. The conductance of a pristine $N_{L}=5$ armchair nanoribbon has been included for comparison (light green dotted line).

It can be observed in the LDOS and in the conductance curves a series of peaks at determined energies. This resonant behavior of the electronic conductance arises from the interference of the electronic wave functions inside the structure, which travel forth and back forming sta-

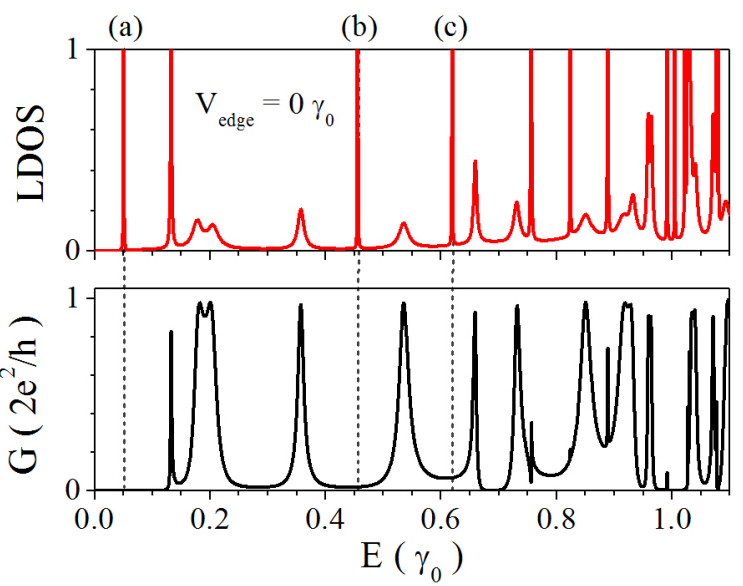

FIG. 2. LDOS (upper panel) and conductance (lower panel) as a function of the Fermi energy for a GQD structure based on leads of width $N_{L}=5$, two symmetric crossbar junctions of width $N_{B}=17$ and $L_{B}=3$. The central region has a width $N_{C}=5$ and length $L_{C}=5$. Marks (a), (b) and (c) denote position of peaks in the LDOS which are absent in the conductance. These states are identified as BICs.

tionary states in the conductor region (well-like states).

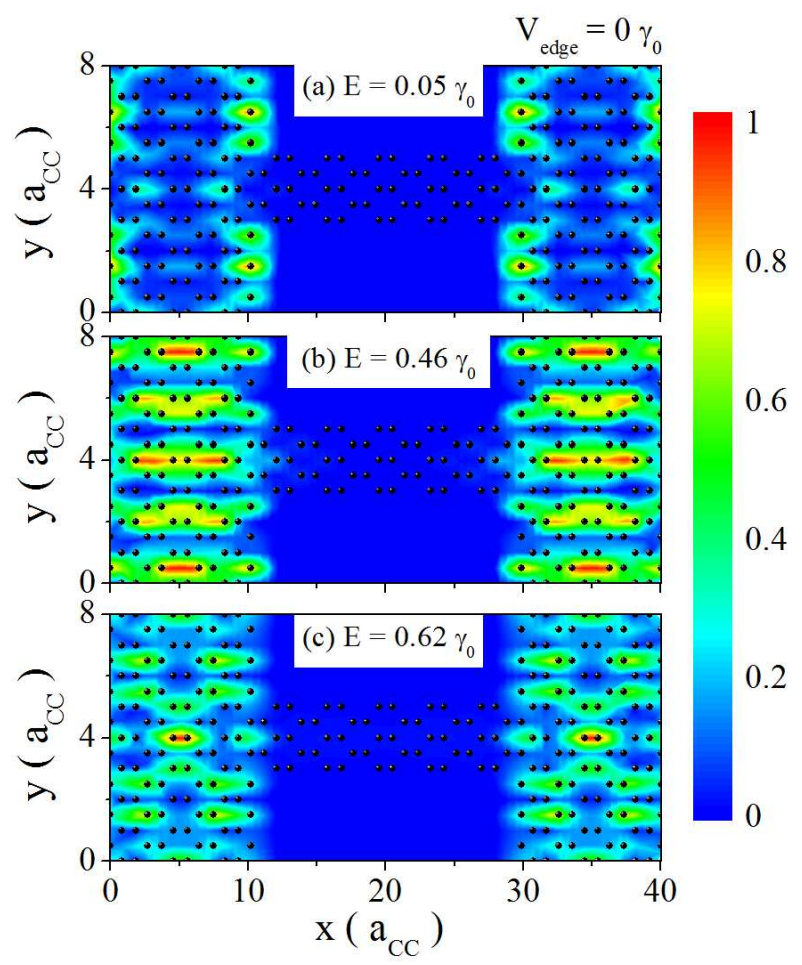

FIG. 3. Corresponding contour plots of some sharp LDOS resonances marked in Fig. 2 LDOS (upper panel).

We start our analysis focusing on the sharp states present in the LDOS displayed in this figure. We have marked the first three sharp states with the letters (a), (b) and (c). We note that the corresponding peaks in 
the conductance are absent and we identify these states as BICs. We calculated the spatial distribution of these states, representing by the corresponding contour plots exhibited in the figure 3. We observe that BICs are completely localized at the region defined by the crossbar junctions. Any electron from the leads, injected at these energies, will be spatially confined in the junctions due to the practically infinite (zero resonance width) lifetime of these states. Therefore BICs do not play any role in the transport properties of these GQD structures. This can be seen in Fig. 2, where the corresponding resonant peaks are absent in the conductance curves.

We note that the bound states in the continuum exhibit a spatial symmetry in the transverse direction of the conductor, with the presence of nodes and maximum in the amplitude of the LDOS along that direction. This behavior is a consequence of the crossbar junction symmetry with respect to the longitudinal axis of the GQD structure. The localized states belonging to the crossbar region could interact between each other through the continuum of states of the pristine ribbon leading to the formation of bound-states in the continuum. In this sense, one of the mechanisms of formation of BICS in our systems correspond to the first one described in the introduction of this letter. Follows the analysis of Moiseyev 32], the number of BICs can be controlled by varying the gate potential applying in the up and down barriers of the GQDs. In order to get a better understanding of this phenomenon, we introduce a simple model that captures the essence of the formation of BICs in our GQD structures. The model consists in two side-coupled impurities attached to a perfect quantum wire [39] as shown in Fig. 4.

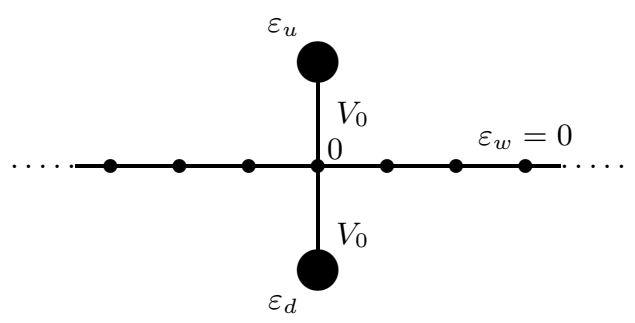

FIG. 4. Scheme of side-coupled impurities attached to a perfect wire.

By using the Dyson equation $G=g+g V G$ we calculate the Green's function $(G)$ in terms of the corresponding Green's function of the isolate sub-systems $(g)$, here $V$ is the matrix coupling between the impurities and the wire $\left(V_{0 u}=V_{0 d}=V_{0}\right)$. To obtain the LDOS of each impurity, $\rho_{\alpha},(\alpha=u, d)$, we calculate the imaginary part of the diagonal elements of the Green's functions, $G_{\alpha}$. Setting the site energies as, $\varepsilon_{u}=\varepsilon_{0}+\delta$ and $\varepsilon_{d}=\varepsilon_{0}-\delta$, $\gamma=$ $\pi V_{0}^{2} \rho(0)$, where $\rho(0)$ corresponds to the LDOS in the site 0 of the wire without impurities, and taking $\delta \ll \gamma$, the density of states of the entire system is obtained summing over $\alpha$ and can be written approximately as,

$$
\rho \approx \frac{1}{\pi} \frac{2 \gamma}{\left(\omega-\varepsilon_{0}\right)^{2}+4 \gamma^{2}}+\frac{1}{\pi} \frac{\delta^{2} / 2 \gamma}{\left(\omega-\varepsilon_{0}\right)^{2}+\left(\delta^{2} / 2 \gamma\right)^{2}} .
$$

The density of states is then the sum of two Lorentzian shapes lines with widths $\Gamma_{+}=2 \gamma$ and $\Gamma_{-}=\delta^{2} / 2 \gamma$, corresponding to those states strongly and weakly coupled to the continuum, respectively. In the limit of $\delta \rightarrow 0$, $\Gamma_{-}$vanishes and the state weakly coupled to the continuum becomes a bound-state in the continuum. This state arises from the interference of the localized states in the impurities, through the continuum states of the wire. In this sense, this interference phenomenon is similar to phenomena like Fano and Aharonov-Bohm effects. If we denote as $\psi_{u}$ and $\psi_{d}$ the wave functions of the up and down impurity respectively, it is straightforward to show that the antisymmetric state, $\left(\psi_{u}-\psi_{d}\right)$ is an eigenstate of the complete system and therefore it is a boundstate in the continuum. In analogy, in the GQD structure

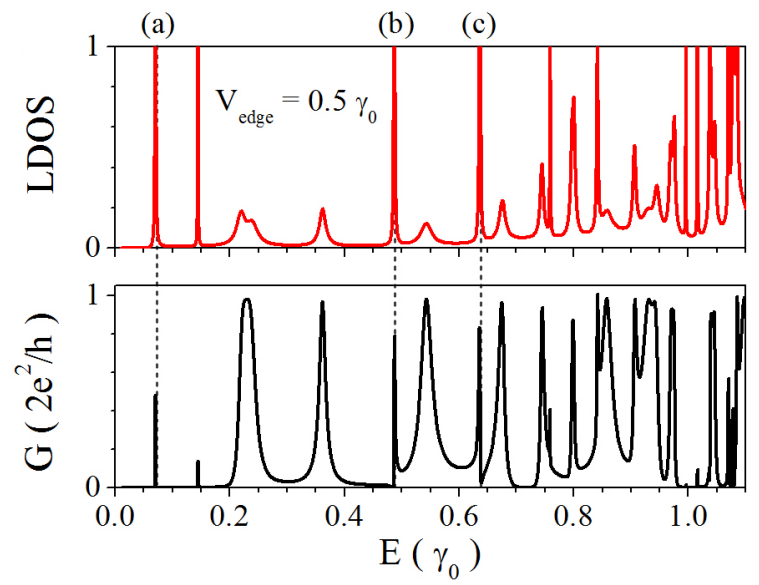

FIG. 5. LDOS (upper panel) and conductance (lower panel) as a function of the Fermi energy for a GQD structure composed by the same parameters of Fig. 2, with an up-down asymmetric gate potential applied to the junction regions.

the formation of the BICs follows the same mechanism. According to it, if any infinitesimal small perturbation breaks the transversal symmetry, the BICs become resonant states with infinitesimal widths. The widths of these "quasi-BICs" can be controlled, for example, by tuning the asymmetry of the system through gate potentials. For instance, figure 5 displays the LDOS and conductance as a function of the Fermi energy, for the same systems considered in figure 2, but now taking into account a small up-down asymmetric gate potential applied to the edges of the GQD. Due to this external perturbation, it is possible to observe the apparition of new peaks of conductance at the BICs energies levels in comparison with the unperturbed case. Therefore, for the feasibility to observe this phenomenon, it is necessary to 
build a GQD as symmetrical as possible and to control the asymmetry via gate potentials. By measuring the changes in the widths of the quasi-BICs in the conductance, it would be possible to obtain indirect evidence of the $\mathrm{BICs}$.

In summary, in this work we have studied the formation of bound-sates in the continuum in quantum-dot like structures. We identify the presence of these states in the LDOS in symmetrical graphene quantum dots structures and we discuss the mechanism of formation of these kind of exotic states. Our results suggests that BICs could be observable in GQDs. The BICs or quasi BICs can have applications in the fields of the spintronics and the quantum computing. For instance, by applying a magnetic field to the GQD structure, the spin degeneracy is lifted and by controlling the resonances width with a gate potential, an efficient spin filter can be designed. On the other hand, the presence of two simultaneous BICs could be used as a qubits for quantum information. Also, BICs could have important applications in photonic crystals, so in this sense, we expect that the study of this kind of states in graphene nanoribbons quantum dots could open a new line of applied research.

The authors acknowledge the financial support of CONICYT/Programa Bicentenario de Ciencia y Tecnología (CENAVA, grant ACT27), USM 110971 internal grant, FONDECYT program grants 11090212, 1100560 and 1100672. L. R. also acknowledges to PUCV-DII grant $123.707 / 2010$.

[*] Electronic address: monica.pacheco@usm.cl

[2] K. S. Novoselov, A. K. Geim, S. V. Morozov, D. Jiang, Y. Zhang, S. V. Dubonos, I. V. Grigorieva, and A. A. Firsov, Science 306, 666 (2004).

[3] C. Berger, Z. Song, T. Li, X. Li, A. Y. Ogbazghi, R. Feng, Z. Dai, A. N. Marchenkov, E. H. Conrad, P. N. First, and W. A. de Heer, J. Phys. Chem. B 108, 19912 (2004).

[4] C. Berger, Z. Song, X. Li, X. Wu, N. Brown, C. Naud, D. Mayou, T. Li, J. Hass, A. N. Marchenkov, E. H. Conrad, P. N. First, W. A. de Heer, Science 312, 1191 (2006).

[5] X. Li, X. Wang, L. Zhang, S. Lee, H. Dai, Science 319, 1229 (2008).

[6] Lijie Ci et al 2008 Nano Res 1116.

[7] D. V. Kosynkin et al . 2009 Nature 458, 872; M. Terrones, 2009 Nature 458845.

[8] S. Stankovich, D. A. Dikin, G. H. B. Dommett, K. M. Kohlhaas, E. J. Zimney, E. A. Stach, R. D. Piner, S. T. Nguyen and R. S. Ruoff, Nature 442, 282 (2006).

[9] F. Schedin, A. Geim, S. Morozov, E. Hill, P. Blake,
M. Katsnelson, K. Novoselov, Nature Materials 6, 652 (2007).

[10] L. Rosales, M. Pacheco, Z. Barticevic, A. Latgé, and P. Orellana, Nanotechnology 19, 065402 (2008).

[11] C. Stampfer, E. Schurtenberger, F. Molitor, J. Gttinger, T. Ihn, and K. Ensslin, Nano Letters 8, 2378 (2008).

[12] J. von Neumann and E. Wigner, Phys. Z. 30, 465 (1929).

[13] Frank H. Stillinger and David R. Herrick, Phys. Rev. A 11, 446 (1975).

[14] H. Friedrich and D. Wintgen, Phys. Rev. A 31, 3964 (1985); H. Friedrich and D. Wintgen, Phys. Rev. A 32, 3231 (1985).

[15] R. L. Schult, H. W. Wyld, and D. G. Ravenhall, Phys. Rev. B 41, 12760 (1990).

[16] Zhen-Li Ji and Karl-Frederik Berggren, Phys. Rev. B 45, 6652 (1992).

[17] J. U. Nöckel, Phys. Rev. B 4615348 (1992).

[18] O. Olendski and L. Mikhailovska, Phys. Rev B 67, 035310 (2003).

[19] I. Rotter and A. F. Sadreev, Phys. Rev. E 71, 046204 (2005).

[20] M.L. Ladrón de Guevara and P.A. Orellana, Phys. Rev. B 73, 205303 (2006).

[21] D.C. Marinica and A.G. Borisov, Phys. Rev. Lett. 100, 183902 (2008).

[22] E. N. Bulgakov and A. F. Sadreev, Phys. Rev. B 78, 075101 (2008).

[23] N Prodanović, V Milanović and J Radovanović J. Phys. A: Math. Theor. 42415304 (2009).

[24] C. Texier, J. Phys. A: Math. Gen. 35, 3389 (2002).

[25] M. Miyamoto, Phys .Rev. A 72, 063405, (2005)

[26] E.N. Bulgakov, I. Rotter and A.F. Sadreev, Phys .Rev. A 75, 067401 (2007).

[27] A.F. Sadreev, E.N. Bulgakov, and I. Rotter, Phys. Rev. B 73, 235342 (2006).

[28] S. Fan, P. R. Villeneuve, J. D. Joannopoulos and H. A. Haus, Phys. Rev. Lett. 80, 960 (1998).

[29] C. S. Kim and A. M. Satanin, Phys. Rev. B 58, 15389 (1998).

[30] E. N. Bulgakov and A. F. Sadreev Phys. Rev. B 81, 115128 (2010).

[31] Federico Capasso, Carlo Sirtori, Jerome Faist, Deborah L. Sivico, Sung-Nee G. Chu and Alfred Y. Cho, Nature 358, 565 (1992).

[32] N. Moiseyev, Phys. Rev Lett. 102, 167404 (2009).

[33] J. W. González, L. Rosales, M. Pacheco, Physica B: Cond. Matt. 404, 2773 (2009).

[34] Young-Woo Son, M. L. Cohen, and S. G. Louie, Phys. Rev. Lett. 97, 216803 (2006).

[35] A. H. Castro Neto, F. Guinea, N. M. R. Peres, K. S. Novoselov, A. K. Geim, Rev. Mod. Phys. 81, 109 (2009).

[36] M. Nardelli, Phys. Rev. B 60, 7828 (1999).

[37] S. Datta, Electronic Transport properties of mersoscopic systems (Cambridge University Press, Cambridge, 1995).

[38] Z.Z. Zhang, Kai Chang and K.S. Chan, Nanotechnology 20, 415203 (2009).

[39] P. Orellana and F. Domínguez-Adame, phys. stat. sol. (a) 203, 1178 (2006). 